\title{
Photon-pair generation in a quadratically nonlinear parity-time symmetric coupler
}

\author{
Diana A. Antonosyan, ${ }^{1}$ Alexander S. Solntsev, ${ }^{1,2}$ and Andrey A. Sukhorukov ${ }^{1, *}$ \\ ${ }^{1}$ Nonlinear Physics Centre, Research School of Physics and Engineering, Australian National University, Canberra, ACT 2601, Australia \\ ${ }^{2}$ School of Mathematical and Physical Sciences, University of Technology Sydney, Ultimo, NSW 2007, Australia \\ ${ }^{*}$ Corresponding author: Andrey.Sukhorukov@anu.edu.au
}

\begin{abstract}
Integrated nonlinear waveguide structures enable generation of quantum entangled photons. We describe theoretically the effects of spatially inhomogeneous loss on the creation of photon pairs through spontaneous parametric down-conversion in quadratically nonlinear directional couplers, where photons experience effective parity-time (PT) symmetric potential due to the presence of optical loss in one of the waveguides. We show that for losses below the PT-breaking threshold, the quantum photon states can be flexibly tuned similarly to conservative couplers, whereas for stronger losses, the correlations between two waveguide modes are suppressed. We also formulate a quantum-classical correspondence with sum-frequency generation for fast evaluation of device performance. These results can be applied for the design of quantum plasmonic circuits.
\end{abstract}

OCIS codes: (190.4410) Nonlinear optics, parametric processes; (190.4390) Nonlinear optics, integrated optics; (080.6755) Systems with special symmetry.

\section{INTRODUCTION}

The development of a new generation of devices for efficient generation of entangled photons is underpinning a range of quantum technologies, including computations, communications, and simulations $[1,2]$. Integrated sources of photon pairs in nonlinear waveguide circuits enable the increase of complexity and enhancement of stability compared to bulk optical implementations (see reviews [3,4] and references therein).

A powerful approach for the control of light propagation in waveguide-based circuits stems from the concept of parity-time (PT) symmetry in optical systems $[5,6]$, which can be implemented with coupled waveguides incorporating symmetrically arranged regions of gain and loss. One of the most interesting effects is the emergence of a phase transition behavior arising from a spontaneous breakdown of PT symmetry, which offers many novel possibilities for shaping optical beams. A broad range of effects qualitatively different from those usually observed in conservative systems has been identified, including power oscillations, nonmonotonous dependence of the transmission on absorption, unidirectional invisibility, conical diffraction, unusual switching regimes, and formation of spatial and temporal solitons in nonlinear structures (see recent reviews $[7,8])$.

Based on the success of PT symmetry in classical photonics, this approach is also likely to be useful for quantum photonic chips, in particular for the generation of spatially entangled photon pairs [4]. Loss is always present in realistic photonic structures, and it has recently been shown that it can enable new possibilities for shaping quantum entangled states in plasmonic circuits [9]. Using the inevitable loss for control of nonlinear photon state generation via PT-symmetry breaking can be a very promising solution. We note that quantum effects have been extensively studied in PT structures with gain and loss, where amplification can lead to both the photon generation and noise [10-13]. In this paper, we explore photon-pair generation in a quadratically nonlinear directional coupler, a structure that has been extensively studied in the non-PT regime with weak homogeneous losses [14]. We consider a regime when material gain is absent, and PT symmetry is introduced purely through loss in one of the waveguides [5,15], which avoids the quantum noise amplification, in contrast to previous studies [10-13]. We show that use of losses and PT-symmetry breaking opens new opportunities for the control of spatially entangled quantum states of the generated photon pairs.

\section{METHODS AND RESULTS}

We study the generation of photon pairs through spontaneous parametric down-conversion (SPDC) in a quadratically nonlinear coupler, as schematically shown in Fig. 1(a). Then, we consider the effect of loss introduced only in the second waveguide, while keeping the first waveguide lossless. Such coupler configuration with no gain realizes PT-symmetric structure up to 


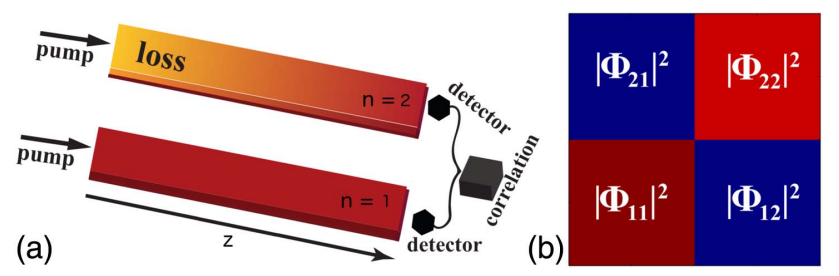

Fig. 1. (a) Scheme of generation of photon pairs through the spontaneous parametric down-conversion in a nonlinear PT-symmetric coupler with linear absorption in the second waveguide. (b) Graphical representation of biphoton correlation function $\left|\Phi_{n_{s}, n_{i}}\right|^{2}$.

a gauge transformation, which can demonstrate all of the key features, including symmetry breaking $[5,15]$. The effect of linear losses on SPDC was previously considered in various contexts [16-18]. We follow the approach of Ref. [19] and formulate Schrödinger-type equations for the photon-pair amplitudes $\Phi_{n_{s}, n_{i}}$ in the coupler as

$$
\begin{aligned}
\frac{\partial \Phi_{n_{s}, n_{i}}(z)}{\partial z}= & -i\left(\beta_{s}+\beta_{i}\right) \Phi_{n_{s}, n_{i}}+\delta_{n_{s}, n_{i}} A_{n_{s}}(z) \\
& -i C_{s} \Phi_{3-n_{s}, n_{i}}-i C_{i} \Phi_{n_{s}, 3-n_{i}} \\
& -\left(\delta_{n_{s}, 2} \gamma_{s}+\delta_{n_{i}, 2} \gamma_{i}\right) \Phi_{n_{s}, n_{i}}
\end{aligned}
$$

where subscripts " $s$ " and " $i$ " refer to the signal and idler photons, $z$ is the propagation distance along the waveguides, $n_{s, i}=1,2$ are the waveguide numbers, $C_{s, i}$ are the coupling constants between the neighboring waveguides, $\beta_{s, i}$ are the propagation constants, $\delta_{n_{s}, n_{i}}$ is the Kronecker delta function, $\gamma_{s, i}$ are the loss coefficients in the second waveguide as we assume there are no losses in the first one, and $A_{n}$ are the classical pump amplitudes at frequency $\omega_{p}$ multiplied by the effective quadratic nonlinearity. We note that absorption of generated photons results in the appearance of single-photon state that can be calculated using the framework of Ref. [19]. Nevertheless, in the regime of a weak pump when four-photon generation probability is small, the two-photon state remains pure even in the presence of loss [18].

In the regime of photon-pair generation, the classical pump remains essentially undepleted, and its evolution is governed by linear coupled-mode equations:

$$
\frac{\partial A_{n_{p}}(z)}{\partial z}=-i \beta_{p} A_{n}-i C_{p} A_{3-n_{p}}-\delta_{n_{p}, 2} \gamma_{p} A_{2}
$$

where $C_{p}$ is the waveguide mode-coupling coefficient, $\beta_{p}$ is the propagation constant, and $\gamma_{p}$ is the loss coefficient in the second waveguide.

We derive the analytical solutions of Eqs. (1) and (2) through a Green's function $[18,20]$, which we define as a solution of linear coupled-mode equations: $\partial G\left(z, n, n^{\prime} ; C, \gamma\right) / \partial z=$ $-i C G\left(z, n, 3-n^{\prime} ; C, \gamma\right)-\delta_{n^{\prime}, 2} \gamma\left(z, n, n^{\prime} ; C, \gamma\right)$ and $G(z=0$, $\left.n, n^{\prime} ; C, \gamma\right)=\delta_{n, n^{\prime}}$. Then, the pump evolution is found as $A_{n_{p}^{\prime}}(z)=\sum_{n_{p}=1}^{2} A_{n_{p}}(0) G\left(z, n_{p}, n_{p}^{\prime} ; C_{p}, \gamma_{p}\right) e^{-i \beta_{p} z}$. We substitute this expression in Eq. (2) and find the biphoton wave function,

$$
\begin{aligned}
\Phi_{n_{s}, n_{i}}(z)= & e^{-i\left(\beta_{s}+\beta_{i}\right) z} \int_{0}^{z} \mathrm{~d} \xi \sum_{n_{p}, n_{p}^{\prime}=1}^{2} A_{n_{p}}(0) \\
& G\left(\xi, n_{p}, n_{p}^{\prime} ; C_{p}, \gamma_{p}\right) G\left(z-\xi, n_{p}^{\prime}, n_{s} ; C_{s}, \gamma_{s}\right) \\
& G\left(z-\xi, n_{p}^{\prime}, n_{i} ; C_{i}, \gamma_{i}\right) e^{i \Delta \beta \xi},
\end{aligned}
$$

where $\Delta \beta=\beta_{s}+\beta_{i}-\beta_{p}$ is the phase mismatch. The form of this solution agrees with the general result for arbitrary photonic structures obtained in Ref. [18].

In the following, we analyze in detail the regime of neardegenerate signal and idler photon frequencies, when accordingly $\gamma_{i}=\gamma_{s}=\gamma$ and $C_{s}=C_{i}=C$ and the antidiagonal elements of the wave function are equal, $\Phi_{1,2}=\Phi_{2,1}$. In the linear propagation regime, both signal and idler photons exhibit PT-symmetry breaking threshold at $\gamma_{\mathrm{PT}}=2 C$, according to the properties of a linear coupler $[5,6]$. Compared to signal and idler, the fundamental pump mode is much stronger localized in a waveguide, resulting in very weak coupling [14]. Additionally, while tailored signal and idler losses can be introduced by depositing metal on top of dielectric waveguides, this will not affect the pump mode. Therefore, we consider $C_{p}=0$ and $\gamma_{p}=0$ in numerical simulations.

Below the critical gain/loss value $\left(\gamma<\gamma_{\mathrm{PT}}\right)$, the photons can periodically tunnel between the coupled waveguides, exhibiting the loss at the effective averaged rate of $\gamma / 2$. As the losses are increased beyond the PT-symmetry breaking point $\left(\gamma>\gamma_{\mathrm{PT}}\right)$, the coupling between the waveguides is suppressed. Then, the mode in the second (lossy) waveguide decays much faster, while the effective loss in the first waveguide is decreased [5].

We now analyze the effect of PT-symmetry breaking on the generation of quantum photon-pair states. For this purpose, we study the signal and idler photon correlations between different waveguides, which are proportional to the squared modulus of the corresponding wave-function elements and can be measured by detecting coincident clicks with single-photon detectors, as schematically illustrated in Fig. 1. Characteristic evolution of correlations along the waveguides is shown in Fig. 2. The top row [Figs. 2(a)-2(c)] corresponds to losses below the PT-breaking threshold. We see that the correlations sensitively depend on the phase mismatch $(\Delta \beta)$, enabling flexible engineering of the two-photon entanglement. In particular, for zero mismatch an N00N-like state with pronounced quantum entanglement is formed, where both signal and idler photons are in superposition of being in the first or second waveguide, but they never appear in different waveguides. In analogy to a conservative nonlinear coupler [14], the maximum correlations between the first and second waveguides are anticipated around phase matching with signal and idler supermodes of the same symmetry, which condition is found using linear supermode dispersion [5] as $\Delta \beta= \pm 2\left[C^{2}-(\gamma / 2)^{2}\right]^{1 / 2}$, providing the values of $\simeq \pm 1.94$ in Figs. 2(a) -2 (c). In sharp contrast, the two-photon state is fundamentally altered when losses exceed the PT-symmetry breaking threshold [see Figs. 2(d)-2(f)]. We observe that the photons are generated by the pump in the first waveguide without loss; however, the photon coupling to the second waveguide is suppressed.

Next, we explore the effect of varying loss in the second waveguide for a fixed propagation distance and present the 


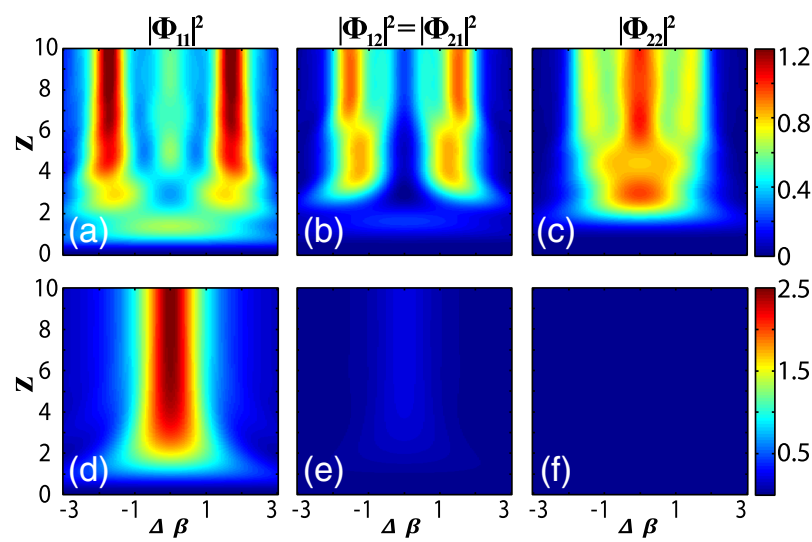

Fig. 2. Evolution of spatial signal and idler photon correlations between the two waveguide modes along the propagation direction (z) depending on the phase mismatch $(\Delta \beta)$ for different losses in the second waveguide $\gamma_{s}=\gamma_{i}=\gamma$. (a)-(c) $\gamma=0.5$ below the PT-symmetry breaking threshold. (d)-(f) $\gamma=3$ corresponding to PT-broken regime. For all plots, the coupling coefficients are $C_{s}=C_{i}=1$, $C_{p}=0$, and pump is coupled to the first waveguide, $A_{1}(z=0)=1$, $A_{2}(z=0)=0$, and $\gamma_{p}=0$.

normalized photon correlations in Fig. 3. When the pump is in the first waveguide without losses [Figs. 3(a)-3(c)], the photonpair correlations in the first waveguide $\left(\left|\Phi_{1,1}\right|^{2}\right)$ dominate when losses in the second waveguide approach and exceed the PTbreaking threshold indicated by the dashed line. For the pump in the second waveguide [Figs. 3(d)-3(f)], the photon pairs can be present in both the first and second waveguides even above the PT-breaking threshold; however, for higher losses, they become dominantly localized in the second waveguide only. We also compare the degree of two-photon entanglement characterized by Schmidt number $Q$ [19]. For the phase mismatch corresponding to stronger correlations between two waveguides, $\Delta \beta=1.5$, and different losses $\gamma=0,1,2,3$, we find that for
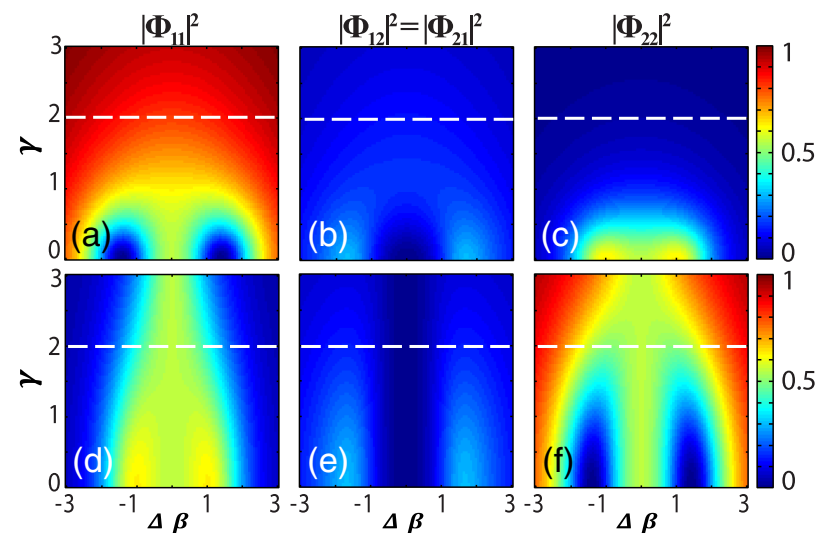

Fig. 3. Normalized photon correlations, $\left|\Phi_{n_{s}, n_{i}}\right|^{2} / \sum_{n_{s}^{\prime}, n_{i}^{\prime}}\left|\Phi_{n_{s}^{\prime}, n_{i}^{\prime}}\right|^{2}$, versus the phase mismatch and loss in the second waveguide at the fixed propagation distance $z=10$. The pump is input to the (a)(c) first $\left[A_{1}(z=0)=1, A_{2}(z=0)=0\right]$ or (d)-(f) second waveguide $\left[A_{1}(z=0)=0, A_{2}(z=0)=1\right]$. Dashed lines indicate the linear PT-breaking threshold at $\gamma_{\mathrm{PT}}=2 C$. Other parameters correspond to Fig. 2. the pump coupled to the first waveguide, entanglement reduces with higher loss as $Q=1.2506,1.0154,1.0024,1.0005$, whereas when the pump is coupled to the second waveguide, the degree of entanglement reaches an intermediate maximum, $Q=1.2506,1.2593,1.3392,1.2539$. Therefore, while the suppression of coupling between the waveguides above the PT-breaking threshold is clearly reflected in photon correlations, the quantitative features of the photon state do nontrivially depend on the input pump configuration and the nonlinear phase mismatch.

The experimental characterization of quantum states requires accumulation of statistics over multiple photon pairs measured with single-photon detectors, which can be time consuming. This motivated the development of approaches for fast classical characterization of nonlinear devices, which then enable one to predict their performance in the regime of quantum photon-pair generation [21,22], including arbitrary multiwaveguide circuits as validated with high experimental fidelity [23]. In the following, we formulate this correspondence for PT nonlinear coupler, considering classical sum-frequency generation (SFG) by the input lasers with the same frequencies as for the signal and idler photons, which are generated in the quantum regime [see Fig. 4(a)]. Then, the SFG is governed by coupled-mode equations,

$$
\begin{gathered}
\frac{\partial u_{n_{s}}}{\partial z}=-i \beta_{s} u_{n_{s}}-i C_{s} u_{3-n_{s}}-\delta_{n_{s}, 2} \gamma_{s} u_{2}+w_{n_{s}} v_{n_{s}}^{*}, \\
\frac{\partial v_{n_{i}}}{\partial z}=-i \beta_{i} v_{n_{i}}-i C_{i} v_{3-n_{i}}-\delta_{n_{i}, 2} \gamma_{i} v_{2}+w_{n_{i}} u_{n_{i}}^{*}, \\
\frac{\partial w_{n_{p}}}{\partial z}=-i \beta_{p} w_{n_{p}}-i C_{p} w_{3-n_{p}}-\delta_{n_{p}, 2} \gamma_{p} w_{2}+u_{n_{p}} v_{n_{p}},
\end{gathered}
$$

where $u, v, w$ are the complex mode amplitudes at the wavelengths corresponding to the signal, idler, and pump wavelengths.

We first consider the regime of relatively weak input amplitudes, when $|w| \ll|u|,|v|$. Accordingly, we employ the undepleted approximation and neglect the last nonlinear terms in Eqs. (4) and (5). Then, these equations become linear, and their solutions can be expressed through a Green's function as $u_{n_{s}^{\prime}}(z)=$ $\sum_{n_{s}=1}^{2} u_{n_{s}}(0) G\left(z, n_{s}, n_{s}^{\prime} ; C_{s}, \gamma_{s}\right) e^{-i \beta_{s} z}$ and $v_{n_{i}^{\prime}}(z)=\sum_{n_{i}=1}^{2} v_{n_{i}}(0)$ $G\left(z, n_{i}, n_{i}^{\prime} ; C_{i}, \gamma_{i}\right) e^{-i \beta_{i} z}$. We then find solution of Eq. (6) as $w_{n_{p}}(z)=e^{-i \beta_{p} z} \int_{0}^{z} \mathrm{~d} \xi \sum_{n_{s}, n_{i}, n_{p}^{\prime}=1}^{2} u_{n_{s}}(0) v_{n_{i}}(0) G\left(\xi, n_{s}, n_{p}^{\prime} ; C_{s}, \gamma_{s}\right)$

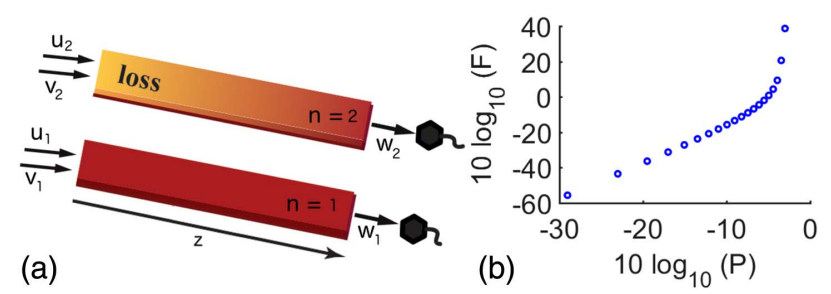

Fig. 4. (a) Scheme of SFG in passive PT-symmetric nonlinear coupler with linear absorption in one waveguide. (b) Mismatch $F$ between the SPDC and SFG depending on the input power $P$ for $\left|u_{n_{\mathrm{s}}}(z=0)\right|^{2}=\left|v_{n_{i}}(z=0)\right|^{2}=P / 2$, plotted on a logarithmic scale. The propagation distance is $z=10$, signal and idler losses are equal $\gamma_{s}=\gamma_{i}=0.5$, there is no loss in the pump mode $\gamma_{p}=0$, and the coupling coefficients are $C_{s}=C_{i}=1, C_{p}=0$. 
$G\left(\xi, n_{i}, n_{p}^{\prime} ; C_{i}, \gamma_{i}\right) G\left(z-\xi, n_{p}^{\prime}, n_{p} ; C_{p}, \gamma_{p}\right) e^{-i \Delta \beta \xi}$. By performing a change of variable $\xi \rightarrow z-\xi$ and using a Green's function symmetry $G\left(z, n^{\prime}, n ; C, \gamma\right) \equiv G\left(z, n, n^{\prime} ; C, \gamma\right)$, we observe the quantum-classical correspondence $w_{n_{p}}(z) \equiv \Phi_{n_{s}, n_{i}}(z)$ when the pumped and detected waveguides are matched in the quantum and classical regimes as $A_{n_{p}}(0)=u_{n_{s}}(0) v_{n_{i}}(0), A_{3-n_{p}}(0)=0$, $u_{3-n_{s}}(0)=0$, and $v_{3-n_{i}}(0)=0$.

While the quantum-classical correspondence is exact in the limit of very weak SFG efficiency, we perform numerical simulations of full Eqs. (4)-(6) and determine the mismatch of the correspondence with SPDC for varying input powers $\left(P=\left|u_{n_{s}}\right|^{2}+\left|v_{n_{i}}\right|^{2}\right)$ in the SFG process, defined as $F=\sum_{n_{s}, n_{i}, n_{p}}\left|w_{n_{p}} /\left[u_{n_{s}}(0) v_{n_{i}}(0)\right]-\Phi_{n_{s}, n_{i}} / A_{n_{p}}(0)\right|^{2}$. The simulation results for the degenerate case $u_{n_{s}}=v_{n_{i}}$ shown in Fig. 4(b) demonstrate that using SFG to predict SPDC in a PT coupler works well until the SFG pump power reaches the values at which it becomes noticeably depleted.

\section{CONCLUSION}

In summary, we predict a possibility to perform flexible control of the quantum state of photon pairs generated in nonlinear coupled waveguides even in the presence of loss, because photon correlations between different waveguides can develop efficiently when operating below the PT-breaking threshold. However, correlations between waveguides get suppressed above the threshold loss value. Furthermore, we formulate a quantum-classical correspondence with SFG for fast evaluation of device performance. These results can facilitate the development of integrated photon sources based on nonlinear plasmonic structures.

Funding. Australian Research Council (ARC) (DP160100619, DE180100070).

\section{REFERENCES}

1. A. Politi, M. J. Cryan, J. G. Rarity, S. Y. Yu, and J. L. O'Brien, "Silicaon-silicon waveguide quantum circuits," Science 320, 646-649 (2008).

2. I. A. Walmsley, "Quantum optics: science and technology in a new light," Science 348, 525-530 (2015).

3. C. L. Xiong, B. Bell, and B. J. Eggleton, "CMOS-compatible photonic devices for single-photon generation," Nanophotonics 5, 427-439 (2016).

4. A. S. Solntsev and A. A. Sukhorukov, "Path-entangled photon sources on nonlinear chips," Rev. Phys. 2, 19-31 (2017).
5. A. Guo, G. J. Salamo, D. Duchesne, R. Morandotti, M. Volatier-Ravat, V. Aimez, G. A. Siviloglou, and D. N. Christodoulides, "Observation of PT-symmetry breaking in complex optical potentials," Phys. Rev. Lett. 103, 093902 (2009).

6. C. E. Ruter, K. G. Makris, R. El Ganainy, D. N. Christodoulides, M. Segev, and D. Kip, "Observation of parity-time symmetry in optics," Nat. Phys. 6, 192-195 (2010).

7. V. V. Konotop, J. K. Yang, and D. A. Zezyulin, "Nonlinear waves in PT-symmetric systems," Rev. Mod. Phys. 88, 035002 (2016).

8. S. V. Suchkov, A. A. Sukhorukov, J. H. Huang, S. V. Dmitriev, C. Lee, and Y. S. Kivshar, "Nonlinear switching and solitons in PT-symmetric photonic systems," Laser Photon. Rev. 10, 177-213 (2016).

9. B. Vest, M. C. Dheur, E. Devaux, A. Baron, E. Rousseau, J. P. Hugonin, J. J. Greffet, G. Messin, and F. Marquier, "Anti-coalescence of bosons on a lossy beam splitter," Science 356, 1373-1376 (2017).

10. G. S. Agarwal and K. N. Qu, "Spontaneous generation of photons in transmission of quantum fields in PT-symmetric optical systems," Phys. Rev. A 85, 031802 (2012).

11. B. He, S. B. Yan, J. Wang, and M. Xiao, "Quantum noise effects with Kerr-nonlinearity enhancement in coupled gain-loss waveguides," Phys. Rev. A 91, 053832 (2015).

12. B. He, L. Yang, and M. Xiao, "Dynamical phonon laser in coupled active-passive microresonators," Phys. Rev. A 94, 031802 (2016).

13. S. Vashahri-Ghamsari, B. He, and M. Xiao, "Continuous-variable entanglement generation using a hybrid PT-symmetric system," Phys. Rev. A 96, 033806 (2017).

14. F. Setzpfandt, A. S. Solntsev, J. Titchener, C. W. Wu, C. L. Xiong, R. Schiek, T. Pertsch, D. N. Neshev, and A. A. Sukhorukov, "Tunable generation of entangled photons in a nonlinear directional coupler," Laser Photon. Rev. 10, 131-136 (2016).

15. M. Ornigotti and A. Szameit, "Quasi PT-symmetry in passive photonic lattices," J. Opt. 16, 065501 (2014).

16. C. M. Caves and D. D. Crouch, "Quantum wide-band traveling-wave analysis of a degenerate parametric-amplifier," J. Opt. Soc. Am. B 4, 1535-1545 (1987).

17. D. Klyshko, Photons and Nonlinear Optics (Gordon \& Breach, 1988).

18. A. N. Poddubny, I. V. Iorsh, and A. A. Sukhorukov, "Generation of photon-plasmon quantum states in nonlinear hyperbolic metamaterials," Phys. Rev. Lett. 117, 123901 (2016).

19. D. A. Antonosyan, A. S. Solntsev, and A. A. Sukhorukov, "Effect of loss on photon-pair generation in nonlinear waveguide arrays," Phys. Rev. A 90, 043845 (2014).

20. W. Vogel and D.-G. Welsch, Quantum Optics, 3rd ed. (Wiley, 2006).

21. M. Liscidini and J. E. Sipe, "Stimulated emission tomography," Phys. Rev. Lett. 111, 193602 (2013).

22. L. G. Helt and M. J. Steel, "Effect of scattering loss on connections between classical and quantum processes in second-order nonlinear waveguides," Opt. Lett. 40, 1460-1463 (2015).

23. F. Lenzini, A. N. Poddubny, J. Titchener, P. Fisher, A. Boes, S. Kasture, B. Haylock, M. Villa, A. Mitchell, A. S. Solntsev, A. A. Sukhorukov, and M. Lobino, "Direct characterization of a nonlinear photonic circuit's wave function with laser light," arXiv: 1703.01007 (2017). 